\title{
Tunable surface roughness and wettability of a soft magnetoactive elastomer
}

\author{
Gašper Glavan ${ }^{1}$, Peter Salamon ${ }^{2}$, Inna A. Belyaeva ${ }^{3}$, Mikhail Shamonin $^{3}$, Irena Drevenšek-Olenik ${ }^{1,4}$ \\ ${ }^{1}$ Faculty of Mathematics and Physics, University of Ljubljana, Jadranska 19, SI1000 Ljubljana, Slovenia \\ ${ }^{2}$ Wigner Research Centre for Physics, Hungarian Academy of Sciences, P.O. Box 49, H-1525 Budapest, Hungary \\ ${ }^{3}$ East Bavarian Centre for Intelligent Materials (EBACIM), Ostbayerische Technische Hochschule Regensburg, \\ Seybothstr. 2, D-93053 Regensburg, Germany \\ ${ }^{4}$ J. Stefan Institute, Jamova 39, SI1000 Ljubljana, Slovenia
}

Correspondence to: I. Drevenšek-Olenik (E-mail: irena.drevensek@ijs.si)

KEYWORDS: magnetorheological elastomers, magnetically responsive surfaces, surface roughness, surface wettability, magnetoactive elastomers

ABSTRACT: Surface topographical modifications of a soft magnetoactive elastomer (MAE) in response to variable applied magnetic field are investigated. The analysis is performed insitu and is based on optical microscopy, spread optical reflection and optical profilometry measurements. Optical profilometry analysis shows that the sensitivity of magnetic fieldinduced surface roughness with respect to external magnetic field is in the range of $1 \mu \mathrm{m} / \mathrm{T}$. A significant hysteresis of surface modifications takes place for increasing and decreasing fields. Investigations of shape of sessile water droplets deposited on the MAE surface reveal that field-induced topographical modifications affect the contact angle of water at the surface. This effect is reversible and the sensitivity to magnetic field is in the range of $20^{\circ} / \mathrm{T}$. Despite the increased surface roughness, the apparent contact angle decreases with 
increasing field, which is attributed to the field-induced protrusion of hydrophilic microparticles from the surface layer.

\section{INTRODUCTION}

Stimuli responsive surfaces are receiving increasing attention in different areas of modern technology. ${ }^{1-4}$ Touch-based interface systems and haptic devices are well-known examples from daily life and mechatronics, respectively. Magnetoactive elastomers (MAEs) are emerging materials for such kind of applications. ${ }^{5-8}$ These composite materials are dispersions of micrometer-sized ferromagnetic particles embedded in a soft elastomer matrix..$^{5,9-15}$ They are distinguished by large modulation of their physical properties (elastic moduli, magneto-mechanical effects, electric properties etc.) in response to external magnetic fields. For instance, recently developed silicone-based MAEs with highly compliant elastomer matrices displayed magnetic field-induced changes of dynamic elastic moduli in the range of three to four orders of magnitude. ${ }^{16,17}$ In the absence of magnetic field, soft MAEs exhibit Young's moduli similar to those of biological tissues (in the order of $10-100$ $\mathrm{kPa}$ ), which consequently makes them promising for applications as magnetically tunable platforms for mediation of biological cell cultures. ${ }^{18}$ Relatively weak magnetic fields $(B<50$ mT) used in these applications are proven to have no direct effect on biological cells, ${ }^{19}$ so the entire mechanism is attributed to modifications of the properties of the supporting medium. In particular, magnetic field-induced stiffening is believed to play a crucial role. ${ }^{18,20}$ However, because cell cultures are deposited on the surface of the cell substrate, it is obvious that they are affected not only by its mechanical properties, but also by its surface properties (e.g. wettability or chemical properties) and surface topography. ${ }^{21,22}$ Magnetic field can also be used for manipulation and control of surface physico-chemical properties of various 
micro-objects. ${ }^{23,24}$ In the context of biomedical applications, it is therefore important to investigate how surface properties of soft MAEs vary as a function of magnetic field.

In contrast to the bulk properties of MAEs, which were investigated in a large number of different studies, ${ }^{5,9-15}$ their surface properties are at present still rather unexplored. Twenty years ago Raphaël and de Gennes theoretically proposed to control the wettability of rubber films by loading them with hard ferromagnetic particles. ${ }^{25}$ The related experimental investigations were performed with rigid polymers, ${ }^{26,27}$ therefore restructuring of the ferromagnetic filler, which is enabled by the soft MAEs, was not possible. It is expected that this restructuring should be noticeable on the free surface of such MAE materials and that the corresponding changes of surface properties should be quite significant.

Most of investigations of MAEs dealing with surface studies were focused on tribological properties. The related analyses of surface topography with scanning electron microscopy (SEM) and atomic force microscopy (AFM) revealed that surface structure was rather uneven and resembled the presence of spherical microparticles within and underneath the surface layer. ${ }^{28-32}$ Recently Lee et al. reported a SEM study of a set of MAE films and showed that surface roughness of samples cured in magnetic field increased with increasing field. ${ }^{33}$ A conventional polydimethylsiloxane (PDMS)-based MAE was used, which at $B \sim 0$ exhibits Young's modulus similar to pure PDMS ( 0.5 MPa).${ }^{34}$ They also demonstrated that magnetic field induces modifications of wetting and adhesion properties of water droplets deposited on uncured films. Different groups demonstrated magnetic control of surface wettability based on manipulation of micropillar surface structures. ${ }^{35-37}$ Very recently Psarra et al. experimentally exploited the stability and post-bifurcation of a non-linear 
system of magnetoelastic film and a substrate block to obtain active control of surface roughness by combining mechanical pre-compression with magnetic-field loading. ${ }^{38}$

In this work, we report experimental study of surface properties of soft MAEs with Young's modulus $<100 \mathrm{kPa}$ (at $B \sim 0)^{39,40}$ Magnetic field plays a role of the stimulus that generates surface modifications. The main novelty of our work is to induce such modifications in the composite with a soft instead of the usual rigid polymer matrix. Optical microscopy, spread optical reflection measurements and optical profilometry were used to investigate variations of surface topography as a function of applied magnetic field. The advantage of optical techniques is, that they can be applied in-situ during modification of magnetic field, because the presence of magnetic field does not disturb the measurement system. The same samples were also used to investigate in-situ magnetic field-induced modifications of the contact angle of sessile water droplets deposited on their surface.

\section{EXPERIMENTAL}

\section{Preparation of soft magnetoactive elastomers}

Fabrication of PDMS-based elastomer material filled with $70 \mathrm{wt} \%$ (corresponding to approximately 22 vol\%) of carbonyl iron powder (CIP, type SQ, BASF SE Carbonyl Iron Powder \& Metal Systems, Ludwigshafen, Germany) took place as reported elsewhere. ${ }^{39,40}$ The initial compound comprised the base polymer VS 100000, the chain extender Modifier 715, the reactive diluent polymer MV 2000, all provided by Evonik Hanse GmbH, Geesthacht, Germany. It was diluted by the silicone oil WACKER ${ }^{\circledR}$ AK 10 (Wacker Chemie AG, Burghausen, Germany). The polymer VS 100000, the polymer MV 2000, the modifier 715 and the silicone oil AK 10 were put together and blended with an electric mixer $\left(\right.$ Roti $^{\circledR}$ Speed-stirrer, Carl Roth $\mathrm{GmbH}$, Germany) to form an initial compound. The mixture 
consisted of VS 100000 (12 g) combined with $2.5 \mathrm{~g}$ of MV 2000, $0.05 \mathrm{~g}$ of Modifier 715 and $33 \mathrm{~g}$ of AK 10. In the next step, the initial compound (12 g) was mixed with CIP (70 \% by mass) and crosslinker 210 (0.036 g). The crosslinking reaction was activated by the PtCatalyst $510(0.024 \mathrm{~g})$. For the activity control of the Pt-catalyst, the inhibitor DVS was employed. The necessary amount of the inhibitor for this MAE composition was $0.012 \mathrm{~g}$.

The incorporated particles had mean diameter of $4.5 \mu \mathrm{m}$ and did not possess any surface coating. No surface treatment was performed before their addition to the initial compound. As described in Ref. 40, the curing process took place in the absence of the external magnetic field, therefore microparticles were uniformly distributed in the polymer matrix (isotropic composite material). ${ }^{9,10,41}$ The fully cured composite sample was cut-out from the film in the form of disk-shaped plates with diameter of $20 \mathrm{~mm}$ and thickness of 2.3 $\mathrm{mm}$. The shear storage modulus of these MAE samples in zero field and in magnetic field of $0.23 \mathrm{~T}$ was measured to be $7.5 \pm 0.4 \mathrm{kPa}$ and $419 \pm 43 \mathrm{kPa}$, respectively. ${ }^{3939}$ Rheological measurements were performed using a commercially available rheometer (Anton Paar, model Physica MCR 301) with the magnetic cell MRD 170/1 T. The angular oscillation frequency $\omega$ was maintained constant at $10 \mathrm{rad} / \mathrm{s}$. To avoid slippage, the normal force of approximately $1 \mathrm{~N}$ was applied. The moduli were measured at constant strain amplitude $\gamma=$ $0.01 \%$, which corresponds to the linear viscoelastic regime. For the sample under investigation, ten measurements of the moduli were performed (measurement time was 20 $s$ per point). The above values of the shear storage modulus are averaged values.

Optical microscopy, spread optical reflectance, optical profilometry and contact angle measurements 
External magnetic field was applied to the sample by placing a NdFeB-type permanent magnet underneath the nonmagnetic sample holder. The dimensions of the magnet were 20 $\times 20 \times 5 \mathrm{~mm}^{3}$. The magnet was mounted on a vertical translation stage and the magnitude of magnetic field in the sample region was varied by changing the distance between the magnet and the sample. The orientation of applied magnetic field in the investigated central region of the sample was perpendicular to the sample surface. Before the experiments, the setup was calibrated by measurement of the field strength at the sample position as a function of translation distance with a Hall sensor. The field was varying in the range from $B=0.05 \mathrm{~T}$ to $B=0.23 \mathrm{~T}$.

Optical polarization microscopy imaging was performed in reflected illumination configuration using a long working distance objective with $5 x$ magnification (Nikon Optiphot2-pol, Nikon, Japan). Spread reflectance measurements were performed with a HeNe laser beam with the wavelength of $633 \mathrm{~nm}$ and output power of $5 \mathrm{~mW}$. The beam was impinging on the sample at the incident angle of $45^{\circ}$. The diameter of the beam on the sample surface was $1 \mathrm{~mm}$. The reflected light was monitored on the opaque screen placed $12.5 \mathrm{~cm}$ behind the sample and imaged by the monochrome camera (IDS UI-3370CP-M-GL, IDS, Germany). The intensity profile of the reflection spot was fitted to the Gaussian function and the obtained FWHM parameter of the fitting curve was used to calculate the effective spreading angle $\theta$ of reflected light with respect to specular reflection direction.

Optical profilometry measurements were performed with a Zygo NewView 7100 3D profiler (Zygo corporation, Middlefield, USA) using a Mirau interferometric objective with 10x magnification. Surface area with size of about $1 \mathrm{~mm} \times 1 \mathrm{~mm}$ was analysed. The profilometer is based on scanning white light interferometry, where the distance between the sample and the interferometric objective is automatically varied, while the 
corresponding micrographs showing the displacement of interference fringes are recorded. The surface topography can be calculated from the data if the lateral resolution allows the unambiguous detection of fringes. At sharp tips, for example, this is not possible, thus there, the instrument does not provide topography data. The obtained data for the height profile $z(x, y)$ were at first flattened via the 3rd order polynomial flattening procedure. Then the resulting profile was further processed to obtain the corresponding root mean square (RMS) roughness and Fourier transform of the surface topography.

Contact angle measurements were performed with a self-constructed setup based on the sessile droplet method using distilled water (Milli-Q system, EMD Millipore corporation, Burlington, USA) doses with the volume of $5 \mu \mathrm{l}$. The experiments took place at temperature of $\sim 22^{\circ} \mathrm{C}$ and relative humidity of $\sim 50 \%$. A video image of the side view of the droplet was captured and afterwards processed to generate its contour line, which was subsequently fitted to a circular shape. Two different fitting parameters were used to calculate the apparent contact angle: (i) the slope of the contour line with respect to the substrate at the contact line (baseline) $\beta$ and (ii) the radius $R$ of the contour line (see inset of Fig. 6). Because of the magneto-deformation effect, the baseline shifts up and down as a function of applied magnetic field, complicating the determination of $\beta$. Therefore, in experiments with variable magnetic field evaluation of contact angle from the values of $R$ was considered to be more reliable.

\section{RESULTS AND DISCUSSION}

\section{Field-induced modifications of surface topography}

In MAEs cured without applied magnetic field, spatial distribution of ferromagnetic microparticles is isotropic in the absence of magnetic field, while in the presence of the field 
particles are rearranged into chain-type aggregates preferentially oriented along the magnetic flux lines. ${ }^{9,10,42,43}$ If the field is oriented perpendicular to the surface, this effect is expected to lead to formation of conical surface structures (designated as "mountains" by Maman and Ponsinet ${ }^{26}$ ) with the top of a "mountain" coinciding with the end of a particular chain-like aggregate (Fig. 1). Such conical structures are well known from magnetorheological liquids, however, the elastomer network of MAEs postures various configurational constraints that result in a more complex topographical restructuring.

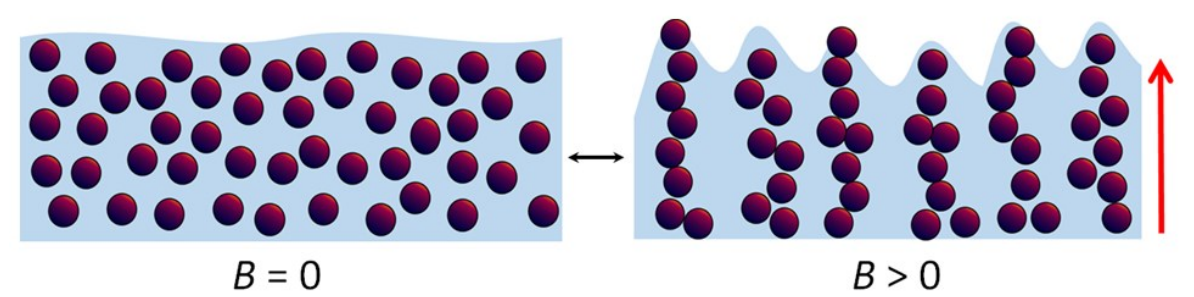

Fig. 1. Schematic drawing of magnetic field-induced modifications of surface morphology of MAEs. Red arrow indicates field direction.

By observing soft MAE samples under ambient light, one can notice that their surface appears smooth and glossy without magnetic field, while it transforms to matte when a magnet is placed below the sample. This signifies field-induced increase of surface roughness. The corresponding topographical modifications were monitored with optical microscopy and the images obtained for three different field magnitudes are shown in Fig. 2. Magnetic field induces a "hills separated by valleys" topography with lateral dimension of the hills in the range of $100 \mu \mathrm{m}$. In addition to these variable structures, some intrinsic fieldindependent imperfections are also present at the surface. 

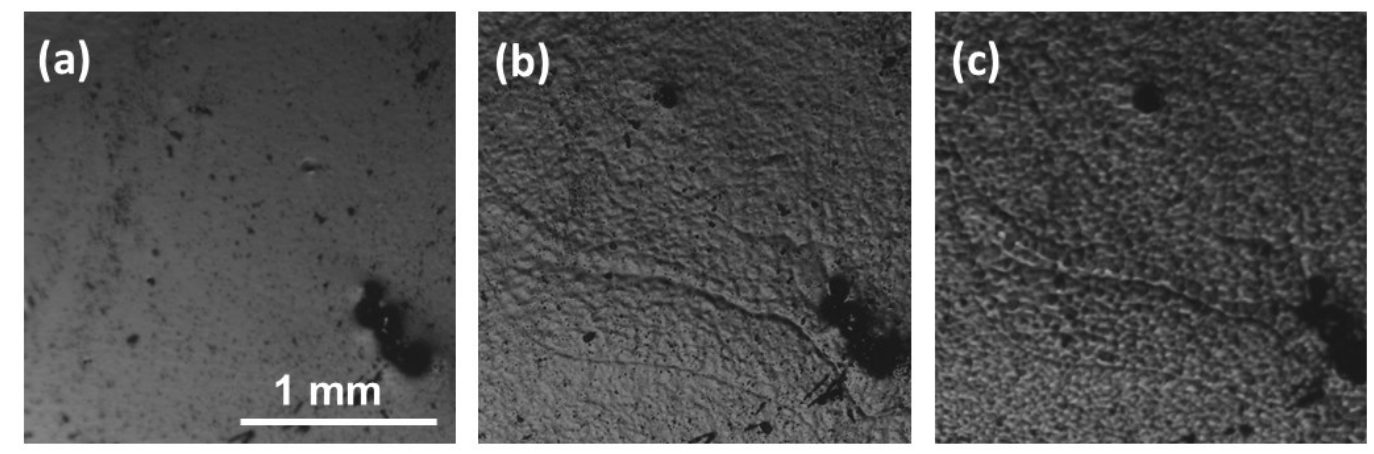

Fig.2 Optical microscopy images of surface topography observed at different magnitudes of applied magnetic field: (a) $B=0.05 \mathrm{~T}$, (b) $B=0.12 \mathrm{~T}$ and (c) $B=0.23 \mathrm{~T}$.

The observed modifications are expected to produce light scattering that increases with increasing field magnitude. When the sample is illuminated with a laser beam, this leads to transition from nearly specular reflection to spread reflection induced by the field. This effect is characterized by an effective cone angle $\theta$ of the spread reflected light that increases with increasing field as shown in Fig. 3a. A significant hysteresis between the behavior obtained for increasing and decreasing fields can be noticed. Similar hysteresis is observed also for field-induced modifications of rheological and other properties of soft MAEs. ${ }^{40,44-46}$

From the obtained values of $\theta(B)$, an estimation of surface roughness can be made by considering a suitable model of surface topography. For this purpose, lateral dimensions of topographical features deduced from optical microscopy images can be taken into account. Because traditional stylus profilers scan the surface in lines, all standardly used roughness parameters are defined with respect to cross sectional profiles of surface topography. In our model, we assume that this profile has a triangular form as depicted in the inset of Fig. 3.b. Based on the lateral structural details deduced from the images shown in Fig. 2, it is 
reasonable to take $L \sim 100 \mu \mathrm{m}$. The RMS roughness of the triangular profile is equivalent to the RMS value of the triangular wave-form, which is ${ }^{47}$

$$
R_{R M S}=\sqrt{\frac{\int_{0}^{L} z(x)^{2} d x}{L}}=\frac{h}{\sqrt{3}}=\frac{(L / 4) \operatorname{tg}(\theta / 2)}{\sqrt{3}},
$$

where $h$ is the amplitude of the profile, $L$ is its lateral period and $\theta / 2$ is the inclination angle of the profile, which is equivalent to the half angle between the incident and the reflected optical beams (for definitions of the parameters see insets in Fig.3). The resulting dependence of $R_{\mathrm{RMS}}(B)$ for increasing magnetic field is shown in Fig. 3.b. To facilitate easier comparison with optical profilometry measurements discussed in the next paragraph (Fig.5.a), the same range of $B$ values is shown as was used in the profilometry experiments. The calculated value of $R_{\mathrm{RMS}}$ is around $0.1 \mu \mathrm{m}$ for $B=0.12 \mathrm{~T}$ and increases to $0.4 \mu \mathrm{m}$ at $B=0.23$ T.

(a)

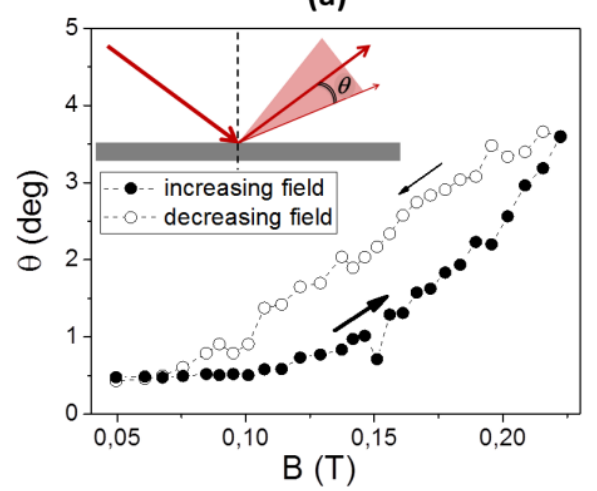

(b)

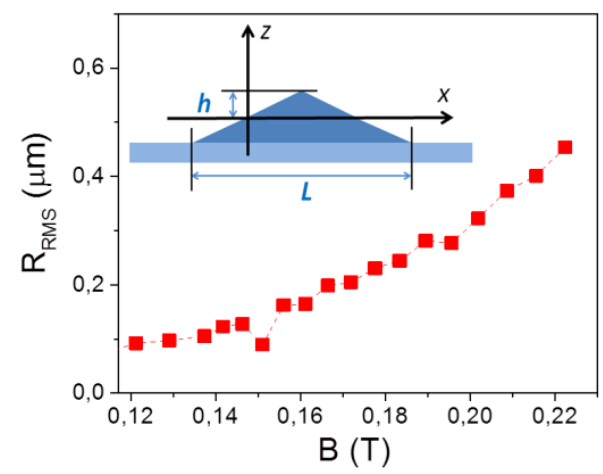

Fig.3 (a) Effective cone angle $\theta$ of spread reflection of laser light as a function of field magnitude. Solid symbols correspond to increasing field and open symbols to decreasing field. (b) Calculated RMS surface roughness for increasing magnetic field. 
Fig. 4 shows 3D optical profilometry images of sample surface observed at $B=0.13 \mathrm{~T}$ and $B=0.23 \mathrm{~T}$, respectively. Due to limited space for vertical translation of the magnet, the minimal field that could have been applied on the sample during profilometric measurements was $0.13 \mathrm{~T}$. To assure that the same surface region was analysed at different field magnitudes, the analysis was performed for a surface region with a characteristic channel that can easily be allocated on the left side of the images. White spots in the images are areas for which the system was unable to generate the image. Similar to optical microscopy (Fig. 2.b,c), also optical profilometry reveals that the height of surface topographical features is notably larger in the larger fields.

(a)

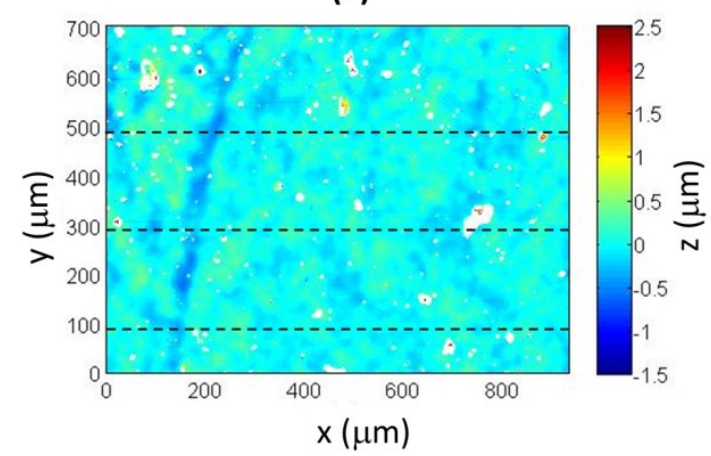

(b)

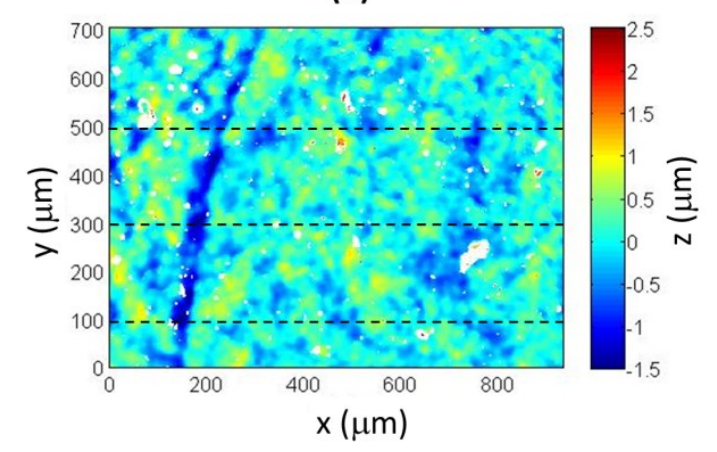

Fig.4 3D surface topography images obtained by optical profilometry measurements at (a) $B=0.13 \mathrm{~T}$ and (b) $B=0.23 \mathrm{~T}$. Cross sectional profiles along the dashed lines are shown in the inset of Fig.5.a.

Further details of field-induced topographical modifications can be resolved in the inset of Fig.5.a, which shows cross sectional profiles $z\left(x, y=y_{0}\right)$ for three different values of $y_{0}$. For clarity, the profiles are vertically shifted for $\Delta z=2 \mu \mathrm{m}$. A significant increase of surface roughness with increasing magnetic field is again evident. Fig.5.a shows magnetic field dependence of the RMS roughness as obtained from the 3D topographical images. The $R_{\mathrm{RMS}}$ is around $0.15 \mu \mathrm{m}$ at $B=0.13 \mathrm{~T}$ and increases towards $0.35 \mu \mathrm{m}$ at $B=0.23 \mathrm{~T}$. These values are in good agreement with the values obtained from spread reflection measurements (Fig.3.b). 
Though, the relative increase of roughness obtained from profilometry data is lower than the one obtained from reflectance analysis. We believe that this is because surface undulation and imperfections differently affect the two experimental methods. For instance, a sharp permanent bump on the surface reflects laser light far away from the specular reflection direction and is consequently not detected as a part of the spread reflection conus, while, on the other hand, it significantly contributes to the measured value of the $R_{\text {RMS. }}$
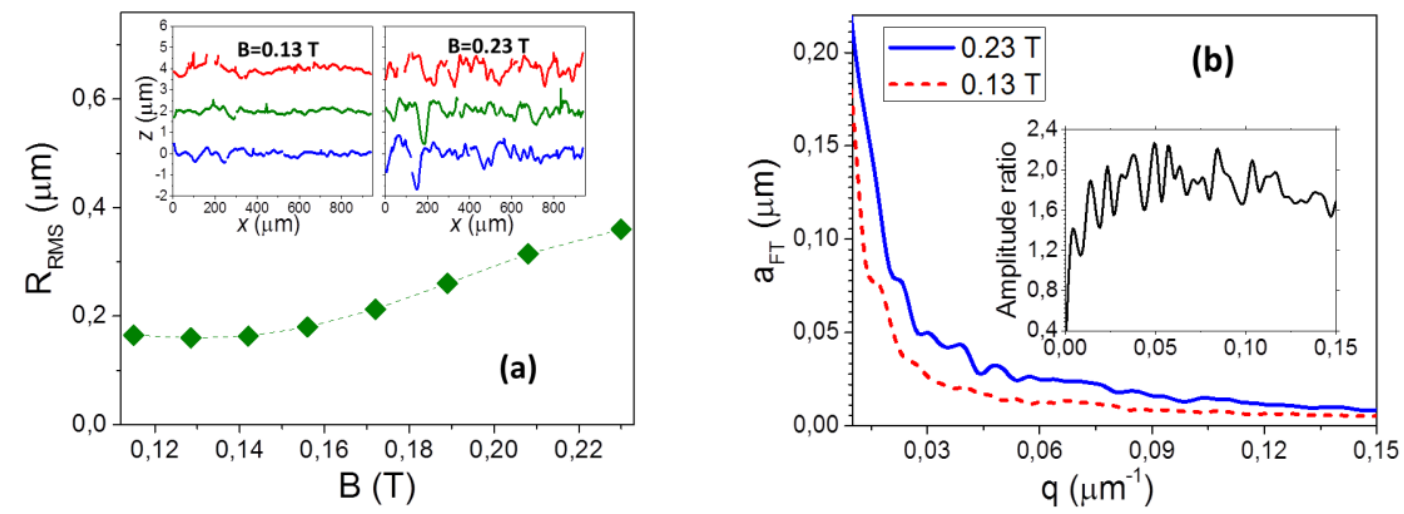

Fig.5 (a) The dependence of RMS roughness resolved from the 3D profilometry images on applied magnetic field. The inset shows topographical cross sections $z(x, y=$ const $)$ along three selected lines at $B=0.13 \mathrm{~T}$ and $B=0.23 \mathrm{~T}$. (b) Azimuthally averaged Fourier transforms of surface topography at $B=0.13 \mathrm{~T}$ (dashed red line) and $B=0.23 \mathrm{~T}$ (solid blue line). The inset shows the ratio of the two curves.

We also performed Fourier transform (FT) analysis of the 3D profiles by applying the twodimensional fast Fourier transform algorithm (in Matlab) on the surface topography data provided by optical profilometry. The resulting amplitude spectra in the Fourier space of wave numbers $\left(q_{x}, q_{y}\right)$ were further processed as follows. In accordance with structural symmetry of the system, the transforms were averaged over azimuthal angles, i.e. the FT amplitude $a_{F T}$ was calculated as a function of the magnitude of the spatial wave vector 
$q=\left(q_{x}^{2}+q_{y}^{2}\right)^{1 / 2}$. The results for obtained for $B=0.13 \mathrm{~T}$ and $B=0.23 \mathrm{~T}$ are shown in Fig. 5.b. One can notice that with increasing field the value of $a_{F T}$ increases for all values of $q$. The inset shows the ratio $a_{F T}(q, B=0.23 \mathrm{~T}) / a_{F T}(q, B=0.13 \mathrm{~T})$. A relatively weak maximum can be noticed at $q_{\max } \sim 0.06 \mu \mathrm{m}^{-1}$, which corresponds to lateral dimension of $\Lambda=2 \pi / q_{\max } \sim 100 \mu \mathrm{m}$, which further supports the model used for analysis of spread reflectance measurements.

\section{Field-induced modifications of contact angle}

The interfacial tension between the water and the substrate is customarily deduced from contact angle measurements. We started our analysis by measurements of contact angle of sessile water droplets during droplet evaporation in a constant applied magnetic field. The results obtained for $B=0.05 \mathrm{~T}$ and $B=0.23 \mathrm{~T}$ are shown in Fig.6. Both resolved properties, the drop inclination with respect to the baseline $\beta$ and its relative radius $R / R_{0}$, where $R_{0}=R(t=0)$, decrease with increasing time. Such behaviour is very common for drop evaporation on micro-textured surfaces and typically proceeds in different stages. ${ }^{48-50}$ The initial value of apparent contact angle $\theta_{\mathrm{c} 0}=\beta(t=0) \sim 105^{\circ}$ is in the range of usual values of contact angle of water observed on pure PDMS. ${ }^{51}$

For $0<t<500 \mathrm{~s}$, the dependencies of $\beta(t)$ and $R(t) / R_{0}$ are almost linear, which is associated with the evaporation stage characterized by a fixed contact line (contact line pinning). ${ }^{49}$ As in our experiments this stage was the most reproducible, all further measurements were performed in this regime. Nevertheless, by repeating experiments with subsequent droplets analysed in the presence of constant applied field of different magnitudes, we could not observe any convincing trends, therefore we decided to investigate the behaviour of one and the same droplet in a variable magnetic field. 


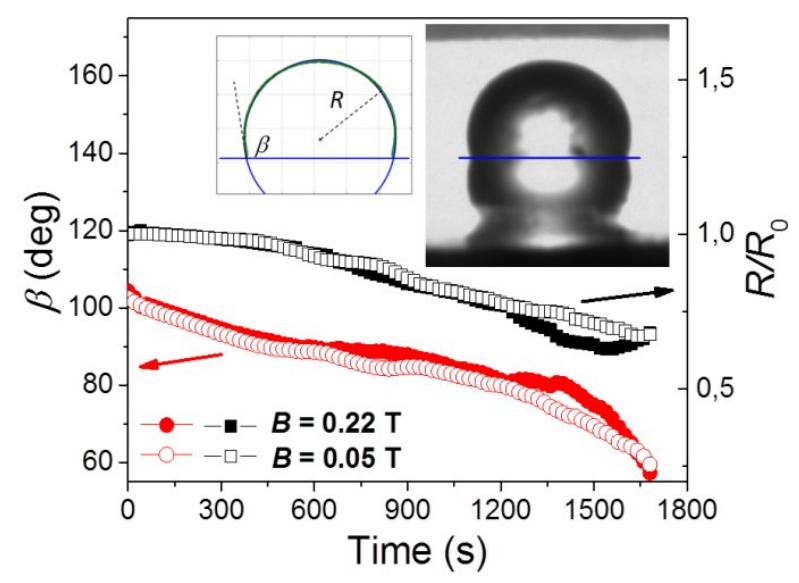

Fig.6 Time dependencies of inclination angle $\beta$ (red symbols) and relative radius of the droplet $R / R_{0}$ (black symbols) during evaporation at two different magnitudes of applied field. The inset on the right shows a photo of the droplet and the inset on the left the corresponding contour line (green curve) and its fit to a circular shape (blue circle).

Fig. 7 shows the results obtained for a transient increase of the applied field. The field was increasing from $B=0.05 \mathrm{~T}$ to $B=0.23 \mathrm{~T}$ during time interval $70 \mathrm{~s}<t<90 \mathrm{~s}$ and decreasing from $B=0.23 \mathrm{~T}$ to $B=0.05 \mathrm{~T}$ during $170 \mathrm{~s}<t<190 \mathrm{~s}$ after deposition of the droplet. Fig.7.a shows the observed dependencies of $\beta(t)$ and $R(t)$, which signify that the apparent contact angle decreases with increasing field. Fig.7.b shows the corresponding modification of the apparent contact angle $\theta_{\mathrm{c}}(B)$ as calculated from the data for $R(t)$. As already mentioned, these data are assumed to be more reliable, because they are not affected by vertical motion of the contact line due to shrinkage (or expansion) of the sample. In calculating $\theta_{c}(B)$ from $R(t)$ we at first rescaled the data to eliminate a decrease of $R$ associated with evaporation. This means that a constant droplet volume was considered when connecting the rescaled values of $R$ with variations of $\theta_{\mathrm{c}}$ induced by magnetic field. The obtained change of the contact angle $\left|\Delta \theta_{\mathrm{c}}\right| \sim 4^{\circ}$ induced by the change of the field from $B=0.05 \mathrm{~T}$ to $B=0.23 \mathrm{~T}$ (and vice versa) is relatively small, i.e. it is in the range of standard deviation of the values of 
$\theta_{\mathrm{c}}$ observed for subsequent droplets at $t=0$, which explains why it is difficult to resolve it in experiments with different droplets.
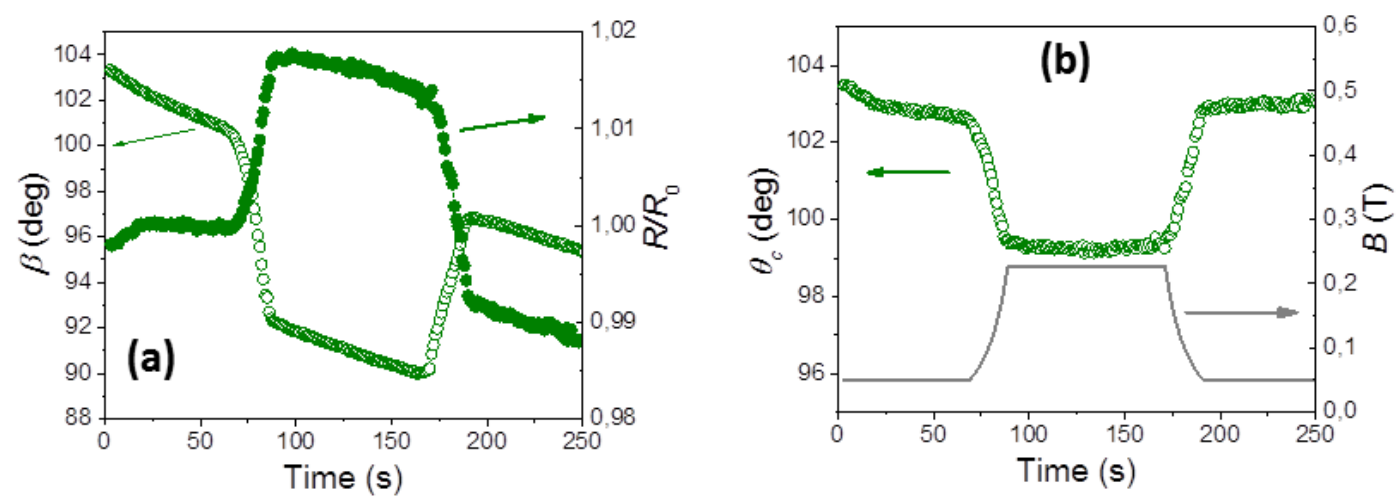

Fig.7 (a) Time dependencies of inclination angle $\beta$ (open symbols) and relative radius of the droplet $R / R_{0}$ (solid symbols) during transient increase of applied magnetic field. (b) Calculated apparent contact angle $\theta_{\mathrm{c}}$ (open symbols) and applied magnetic field (solid line) as a function of time.

Similar analysis was performed also for a transient decrease of magnetic field. The results are shown in Fig. 8. In this case the contact angle temporary increases. The obtained modification is $\left|\Delta \theta_{\mathrm{c}}\right| \sim 3^{\circ}$.
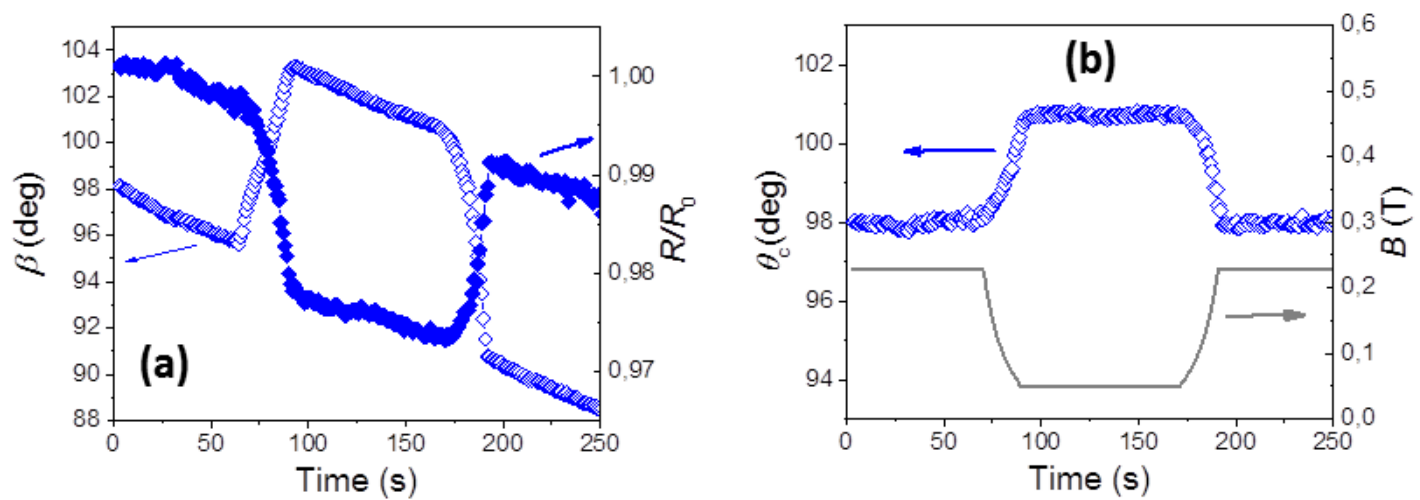

Fig.8 (a) Time dependencies of inclination angle $\beta$ (open symbols) and relative radius of the droplet $R / R_{0}$ (solid symbols) during transient decrease of applied magnetic field. (b) Calculated apparent contact angle $\theta_{\mathrm{c}}$ (open symbols) and applied magnetic field (solid line) as a function of time. 
Our experiments show that the contact angle of water on the surface of our samples decreases with increasing magnetic field despite the fact that their surface roughness increases. This seems quite contradictory, since for hydrophobic surfaces $\left(\theta_{c 0}>90^{\circ}\right)$, both, the Wenzel and the Cassie-Baxter model that are conventionally used to explain wetting phenomena on microstructured surfaces, predict the opposite effect. ${ }^{52,53}$ Correspondingly, also in the combined model described by

$$
\cos \theta_{c}=r_{s} f \cos \theta_{c 0}-(1-f),
$$

where $r_{\mathrm{s}}$ is a roughness factor (defined as a ratio between the wetted surface area and its projection to a $2 \mathrm{D}$ plane) and $f$ is the fraction of surface area that is in contact with water (the rest is in contact with trapped air pockets), leads to $\theta_{\mathrm{c}}>\theta_{\mathrm{c} 0}$ for any value of $f$.

For our samples the lateral extension of topographical objects defining the roughness is in the range of $100 \mu \mathrm{m}$, while their height is in the range of $1 \mu \mathrm{m}$, thus the values of $r_{\mathrm{s}}$ are very small. From 3D optical profilometry images we calculated $r_{\mathrm{f}}=1.001$ for $B=0.12 \mathrm{~T}$ and $r_{\mathrm{f}}$ $=1.002$ for $B=0.23 \mathrm{~T}$. This means that in view of standard experimental accuracy of contact angle measurements, the Wenzel effect is completely negligible and $r_{\mathrm{s}}=1$ can be used in Eq.2. Due to smoothness of the topographical details (see inset of Fig.5.a) also formation of air pockets between the droplet and the surface is not very probable, so it is reasonable to take also $f \sim 1$.

These conclusions suggest that not roughness modifications, but some other fieldinduced effect is responsible for the observed wetting changes. We propose that this is a modification of surface chemical composition due to magnetic field-induced protrusion of microparticles from bulk to the surface (Fig. 1.b). Such protrusion was recently observed in 
conventional MAE materials, ${ }^{33}$ so in the soft MAEs it is expected to be even more profound. For $B \sim 0$, microparticles are positioned underneath the surface and consequently the top surface layer is composed of PDMS, while for $B>0$, due to formation of field-induced chainlike aggregates, microparticles are partially pushed out from the surrounding matrix and become involved in the surface interaction processes (Fig. 1). To take into account the corresponding modification of chemical composition, the Cassie-Baxter model can be used in the form: ${ }^{52}$

$$
\cos \theta_{c}=\left[f_{p}(B) \cos \theta_{c p}+\left(1-f_{p}(B)\right) \cos \theta_{c 0}\right],
$$

where $f_{\mathrm{p}}(B)$ is a relative contact area of the droplet with protruded particles, which is a function of $B$, and $\theta_{\mathrm{cp}}$ is the contact angle of water on the surface of particles. If $\theta_{\mathrm{cp}}>\theta_{\mathrm{co}}$, microparticles protrusion promotes the hydrophobicity and consequently $\theta_{\mathrm{c}}>\theta_{\mathrm{c} 0}$, while for $\theta_{\mathrm{cp}}<\theta_{\mathrm{c} 0}$ it promotes hydrophilicity and hence $\theta_{\mathrm{c}}<\theta_{\mathrm{c} 0}$. By assuming complete wetting of microparticle surface $\left(\theta_{\mathrm{cp}}=0\right)$ and linear increase of their contact area with increasing magnetic field $\left(f_{\mathrm{p}}(B)=k B\right)$ it follows: ${ }^{52}$

$$
\cos \theta_{c}=k B\left(1-\cos \theta_{c 0}\right)+\cos \theta_{c 0},
$$

where $k$ is a proportionality constant. For the field-dependent contact angle values observed in our experiments, namely $\theta_{c}(B=0.05 \mathrm{~T})=102^{\circ} \pm 0.5^{\circ}$ and $\theta_{c}(B=0.23 \mathrm{~T})=98.5^{\circ} \pm 0.5^{\circ}$, Eq.4 gives $\theta_{\mathrm{c} 0}=103^{\circ} \pm 1^{\circ}$ and $k=0.27 \pm 0.07 \mathrm{~T}^{-1}$. This means that at $B=0.23 \mathrm{~T}$ about $6 \%$ of surface contact area is transformed from PDMS to carbonyl iron. To obtain an approximate value of $\theta_{\mathrm{cp}}$ for uncoated carbonyl iron microparticles that were used for our samples, we grafted a layer of 
these particles onto a sticky substrate and deposited several water droplets over it. We observed practically complete wetting behaviour, therefore taking $\theta_{\mathrm{cp}}=0$ is reasonable. On the other hand, if silanized particles are used, one should take $\theta_{\mathrm{cp}}=180^{\circ}$. Therefore, by appropriate selection of microparticle surface coating one can regulate the effect either towards increased hydrophobicity or hydrophilicity. ${ }^{54,55}$ The magnetic field-induced contact angle modifications observed in our experiments $\left(\Delta \theta_{c} \sim 4^{\circ}\right)$ are considerably smaller than the ones observed by Lee et al. in experiments with silanized microparticles $\left(\Delta \theta_{c} \sim 60^{\circ}\right),{ }^{33}$ which is attributed to the fact that they used uncured (liquid state) MAE mixtures.

\section{CONCLUSIONS AND FURTHER PERSPECTIVES}

Our results demonstrate that surface roughness of soft MAEs can be dynamically tuned by variation of applied magnetic field. The tunability observed in our samples is in the range of $\Delta R_{\mathrm{RMS}} / \Delta B \sim 1 \mu \mathrm{m} / \mathrm{T}$ but this value can very probably be increased by optimization of the material composition. Further experiments with materials involving different concentrations of microparticles and different softness of elastomer network are needed to find the optimal formulation. We also showed that field-induced surface changes exhibit similar hysteresis as bulk elastic properties, which signifies that they are coupled to each other. The hysteresis provides bistability and memory functions in the form of surface topographical modifications. We also showed that an increase of surfaces roughness of MAEs does not necessarily play a dominant role in the associated modification of wetting properties, as also field-induced protrusion of microparticles from the bulk to the surface can be very important. Also this phenomenon needs to be further investigated to resolve the details of the associated features and enable its further optimization. 
Our results show that the wettability of MAE surface can be tuned by magnetic field. All our experiments were limited to analysis of sessile droplets in the initial stage of evaporation process, but, it is quite probable, that in other stages of the evaporation process, fieldinduced modifications of the contact angle would behave differently. Besides this, filled elastomeric materials undergoing repeated deformation cycles often present a certain degree of 'conditioning', which can be associated with the hysteresis in their mechanical response. ${ }^{56}$ This effect might cause a progressive decrease of the response of the surface microstructure over time and consequently affect its wettability. For example, the Mullins effect is known in MAEs. ${ }^{57}$ The Mullins effect (cyclic stress softening) is defined as an instantaneous and irreversible softening of the stress-strain curve that occurs whenever the load increases beyond its prior all-time maximum value. ${ }^{58}$ However, the Mullins effect is usually expected at much larger deformations ${ }^{59}$ than those caused by magnetic fields in the present paper. One should also test contact angle hysteresis as a function of applied field, as well as the effect of applied field on sliding angle of the droplets. An intriguing challenge is, for instance, to use magnetic field-induced surface changes for switching between adhesive and sliding regime of the droplets.

\section{ACKNOWLEDGEMENTS}

This work was performed in the framework of the Slovenian research program P1-092 ("Light and Matter"). The authors acknowledge financial support of the joint research project between Slovenian Academy of Sciences and Arts and Hungarian Academy of Sciences ("Microfluidic systems based on anisotropic soft matter") and support by the Slovenian Research Agency (SRA) and the German Academic Exchange Service (DAAD) of the bilateral project "Magnetically reconfigurable elastomeric optical surfaces" (SRA project no. 
BI-DE/17-19-17, DAAD project-ID 57364932) for realization of exchange visits. Furthermore, the financial support from the National Research, Development and Innovation Office (NKFIH, Hungary) Grant No. FK 125134 and PD 121019 are gratefully acknowledged. We thank Aladár Czitrovszky for the access to the profilometer.

\section{REFERENCES AND NOTES}

1. Stuart, M. A. C.; Huck, W. T.; Genzer, J.; Müller, M.; Ober, C.; Stamm, M., ... \& Winnik, F. Nat. Mater. 2010, 9, 101.

2. Nath, N.; Chilkoti, A. Adv. Mater. 2002, 14, 1243.

3. Mendes, P. M. Chem. Soc. Rev. 2008, 37, 2512.

4. Huang, X.; Sun, Y.; Soh, S. X. Adv. Mater. 2015, 27, 4062.

5. Ubaidillah; Sutrisno, J.; Purwanto, A.; Mazlan, S. A. Adv. Eng. Mater. 2015, 17, 563.

6. Kikuchi, T.; Masuda, Y.; Sugiyama, M.; Mitsumata; T.; Ohori, S. J. Phys. Conf. Ser. 2013, 412, 012036.

7. Böse, H. In Magnetorheological elastomers with continually adjustable hardness, available at: https://www.cesma.de/content/dam/isc/cesma/de/documents/3 MR Elastomers 2013 .pdf

8. Hatzfeld, C.; Bilz, J.; Fritzsche, T.; Kupnik, M. In International Conference on Human Haptic Sensing and Touch Enabled Computer Applications, Springer International Publishing, 2016, pp 109-119.

9. Filipcsei, G.; Csetneki, I.; Szilágyi, A.; Zrínyi, M. Adv. Polym. Sci. 2007, 206, 137.

10. Abramchuk, S.; Kramarenko, E.; Stepanov, G.; Nikitin, L. V.; Filipcsei, G.; Khokhlov, A. R.; Zrinyi, M. Polym. Adv. Technol. 2007, 18, 883.

11. Li, Y.; Li, J.; Li, W.H. Du, H. Smart Mater. Struct. 2014, 23, 123001.

12. Menzel, A. M. Phys. Reps. 2015, 554, 1.

13. Odenbach, S. Arch. Appl. Mech. 2016, 86, 269.

14. Lopez-Lopez, M.; Durán, J. D.; Iskakova, L. Y.; Zubarev, A. Y. J. Nanofluids 2016, 5, 479.

15. Cantera, M. A.; Behrooz, M.; Gibson, R. F.; Gordaninejad, F. Smart Mater. Struct. 2017, 26, 023001.

16. Chertovich, A. V.; Stepanov, G. V.; Kramarenko, E. Y.; Khokhlov, A. R. Macromol. Mater. Eng. 2010, 295, 336. 
17. Stoll, A.; Mayer, M.; Monkman, G. J.; Shamonin, M. J. Appl. Polym. Sci. 2014, 131, 39793.

18. Mayer, M.; Rabindranath, R.; Börner, J.; Hörner, E.; Bentz, A.; Salgado J. et al., PLoS ONE 2013, 8, e76196.

19. Campos Albuquerque, W. W.; Brandão Costa, R. M. P.; de Salazar e Fernandes, T.; Figueiredo Porto, A. L. Prog. Biophys. Mol. Biol. 2016, 121, 16.

20. Han, Y.; Hong, W.; Faidley, L. E. Int. J. Solids. Struct. 2013, 50, 2281.

21. Rosales-Leal, J. I.; Rodríguez-Valverde, M. A.; Mazzaglia, G.; Ramón-Torregrosa, P. J.; Díaz-Rodríguez, L.; García-Martínez, O. O.; Vallecillo-Capilla, M.; Ruiz, C.; Cabrerizo-Vílchez, M. A. Colloids Surf A Physicochem Eng Asp. 2010, 365, 222.

22. Dhowre, H. S.; Rajput, S.; Russell, N. A.; Zelzer, M. Nanomedicine 2015, 10, 849.

23. Delcea, M.; Moehwald, H.; Skirtach, A. G. Adv. Drug Deliv. Rev. 2011, 63, 730.

24. Gorin, D. A.; Portnov, S. A.; Inozemtseva O. A.; Luklinska, Z.; Yashchenok, A. M.; Pavlov, A. M.; Skirtach, A. G.; Moehwald, H.; Sukhorukov, G. B. Phys. Chem. Chem. Phys. 2008, 10, 6899.

25. Raphael, E.; De Gennes, P.-G. C.R. Acad. Sci. Paris Série I/ b Surfaces, Interfaces, Films. 1997, 325, 537 (in French).

26. Maman, M.; Ponsinet, V. Langmuir 1999, 15, 259.

27. Nagappan, S.; Ha, C. S. J. Mat. Chem. 2015, 3, 3224.

28. Li, R.; Ren, D.; Wang, X.; Chen, X.; Wu, X. J. Intell. Mater. Syst. https://doi.org/10.1177/1045389X17708043

29. Wang, K.; Gao, W.; Wang, X.; He, H. J. Appl. Polym. Sci. 2017, 134, 44559.

30. Chen, D.; Yu, M.; Zhu, M.; Qi, S.; J. Fu, Smart. Mater. Struct. 2016, 25, 115005.

31. Lian, C.; Lee, K.-H.; An, J.-W; Lee, C.-H Friction 2017, 5, 2017 https://doi:10.1007/s40544-017-0150-1

32. Iacobescu, G. E.; Balasoiu, M.; Bica, I. J. Supercond. Nov. Magn. 2013, 26, 785.

33. Lee, S.; Yim, C.; Kim, W.; Jeon, S. ACS Appl. Mater. Interfaces 2015, 7, 19853.

34. Lee, D.; Lee, M.; Jung, N.; Yun, M.; Lee, J.; Thundat, T.; Jeon, S. Smart. Mater. Struct. 2014, $23,055017$.

35. Zhou, Q.; Ristenpart, W. D.; Stroeve, P. Langmuir 2011, 27, 11747.

36. Zhu, Y.; Antao, D. S.; Xiao, R.; Wang, E. N. Adv. Mater. 2014, 26, 6442.

37. Drotlef, D. M., Blümler, P.; Papadopoulos, P.; del Campo, A. ACS Appl. Mater. Interfaces 2014, 6, 8702.

38. Psarra, E.; Bodelot, L.; Danas, K. Soft Matter 2017, 13, 6576. 
39. Sorokin, V. V.; Belyaeva, I. A.; Shamonin, M.; Kramarenko, E. Yu. Phys. Rev. E 2017, 95, 062501.

40. Belyaeva, I. A.; Kramarenko, E. Y.; Shamonin, M. Polymer 2017, 127119.

41. Agirre-Olabide, I.; Lion, A.; Elejabarrieta, M. J. Smart. Mater. Struct. 2017, 26, 035021.

42. An, H.-N.; Picken, S. J.; Mendes, E. Soft Matter 2012, 8, 11995.

43. Borbath, B. T.; Günther, S.; Borin, D. Yu.; Gundermann, Th.; Odenbach, S. Smart Mater. Struct. 2012, 21, 105018.

44. Sorokin, V. V.; Stepanov, G. V.; Shamonin, M.; Monkman, G. J.; Khokhlov A. R.; Kramarenko, E. Y. Polymer 2015, 76, 191

45. Biller, A.; Stolbov O.; Raikher, Y. L. Phys. Rev. E 2015, 92, 023202.

46. Zubarev, A. Y.; Chirikov, D. N.; Borin, D. Y.; Stepanov, G. V. Soft Matter 2016, 12, 6473.

47. https://en.wikipedia.org/wiki/Root_mean_square, November 2017.

48. Chen, X.; Ma, R.; Li, J.; Hao, C.; Guo, W.; Luk, B.L.; Li, S. C.; Yao, S.; Wang, Z. Phys. Rev. Lett. 2012, 109, 116101.

49. Yu, D. I.; Kwak, H. J.; Doh, S. W.; Kang, H. C.; Ahn, H. S.; Kiyofumi, M.; Park, H. S.; Kim, M. H. Int. J. Heat Mass Transfer 2015, 90, 191.

50. Ramos, S. M. M.; Dias, J. F.; Canut, B. J. Colloid Interface Sci. 2015, 440, 133.

51. Cortese, B.; D’Amone, S.; Manca, M.; Viola, I.; Cingolani, R.; Gigli, G. Langmuir 2008, 24, 2712.

52. Bormashenko, E. Adv. Colloid Interface Sci. 2015, 222, 92.

53. Marmur, A. Soft Matter 2006, 2, 12.

54. Yilgor, I.; Bilgin, S.; Isik, M.; Yilgor, E. Langmuir 2012, 28, 14808.

55. Cvek, M.; Moucka, R.; Sedlacik, M.; Pavlinek, V. Smart. Mater. Struct. 2017, 26, 105003.

56. Bergström, J. S.; Boyce, M. C. Rubber Chem. Technol. 1999, 72, 633.

57. Coquelle, E.; Bossis, G. Int. J. Solid Struc. 2006, 43, 7659.

58. Diani, J.; Fayolle, B.; Gilormini, P. Eur. Polym. J. 2009, 45, 601.

59. Cantournet, S.; Desmorat, R.; Besson, J. Int. J. Solid Struc. 2009, 46, 2255. 


\section{FIGURE CAPTIONS}

Fig. 1. Schematic drawing of magnetic field-induced modifications of surface morphology of MAEs. Red arrow indicates field direction.

Fig.2 Optical microscopy images of surface topography observed at different magnitudes of applied magnetic field: (a) $B=0.05 \mathrm{~T}$, (b) $B=0.12 \mathrm{~T}$ and (c) $B=0.23 \mathrm{~T}$.

Fig.3 (a) Effective cone angle $\theta$ of spread reflection of laser light as a function of field magnitude. Solid symbols correspond to increasing field and open symbols to decreasing field. (b) Calculated RMS surface roughness for increasing magnetic field.

Fig.4 3D surface topography images obtained by optical profilometry measurements at (a) $B=0.13 \mathrm{~T}$ and $(\mathrm{b}) B=0.23 \mathrm{~T}$. Cross sectional profiles along the dashed lines are shown in the inset of Fig.5.a.

Fig.5 (a) The dependence of RMS roughness resolved from the 3D profilometry images on applied magnetic field. The inset shows topographical cross sections $z(x, y=$ const $)$ along three selected lines at $B=0.13 \mathrm{~T}$ and $B=0.23 \mathrm{~T}$. (b) Azimuthally averaged Fourier transforms of surface topography at $B=0.13 \mathrm{~T}$ (dashed red line) and $B=0.23 \mathrm{~T}$ (solid blue line). The inset shows the ratio of the two curves. 
Fig.6 Time dependencies of inclination angle $\beta$ (red symbols) and relative radius of the droplet $R / R_{0}$ (black symbols) during evaporation at two different magnitudes of applied field. The inset on the right shows a photo of the droplet and the inset on the left the corresponding contour line (green curve) and its fit to a circular shape (blue circle).

Fig.7 (a) Time dependencies of inclination angle $\beta$ (open symbols) and relative radius of the droplet $R / R_{0}$ (solid symbols) during transient increase of applied magnetic field. (b) Calculated apparent contact angle $\theta_{c}$ (open symbols) and applied magnetic field (solid line) as a function of time.

Fig.8 (a) Time dependencies of inclination angle $\beta$ (open symbols) and relative radius of the droplet $R / R_{0}$ (solid symbols) during transient decrease of applied magnetic field. (b) Calculated apparent contact angle $\theta_{c}$ (open symbols) and applied magnetic field (solid line) as a function of time. 